Pacific Journal of Mathematics

HOMEOMORPHISM GROUPS OF DENDRONS 


\title{
HOMEOMORPHISM GROUPS OF DENDRONS
}

\author{
Beverly L. Brechner
}

Professor J. Dugundji has asked the following question. Is the space of homeomorphisms of a contractible, locally contractible, space necessarily locally contractible? In this paper we answer the question in the negative by showing that the spaces of homeomorphisms of certain dendrons are not locally contractible. Specifically, we will show that any "special" dendron (see Definition 2.3) has a zero dimensional, non-discrete, group of homeomorphisms.

Conventions and notation. If $X$ is a metric continuum, $G(X)$ will denote the group of all homeomorphisms of $X$, with the following metric topology:

$$
d(g, h)=\operatorname{lub}_{x \in X}\{d(g(x), h(x))\} .
$$

The double arrow in $f: A \rightarrow B$ means onto.

By a dendron is meant a locally connected metric continuum containing no simple closed curves.

See [3] and [4] for further discussion of dendrons.

\section{Homeomorphisms between similar dendrons.}

Definition 2.1. $A$ set $B$ is an order basis for a dendron $D$ if and only if every point $x$ of $D$ has arbitrarily small neighborhoods $U_{x}$, with boundaries in $B$, such that the number of points on $\mathrm{Bd} U_{x}$ is less than or equal to the order of $x$ [3, p. 277].

Definition 2.2. Let $D$ be a dendron, $B$ an order basis for $D$. Let $\mathscr{Y}: W_{1}, \cdots, W_{n}$ be a cover of $D$ of mesh $<\varepsilon$ with the following properties for each $i$ :

(1) $W_{i}$ is a continuum which is the closure of an open set,

(2) $\mathrm{Bd} W_{i}$ is a finite subset of $B$, and

(3) $W_{i} \cap W_{j}=\mathrm{Bd} W_{i} \cap \mathrm{Bd} W_{j}$ for each $j \neq i$. Then $\mathscr{W}$ is called a regular $\varepsilon$-cover with respect to $B$.

Definition 2.3. Let $S$ be a nonempty set, finite or infinite, of positive integers greater than or equal to three. Let $\mathscr{D}_{S}$ be the class of all dendrons with a dense set of cut points of order $n$, for each $n \in S$, and no cut points of order $k \geqq 3$, if $k \notin S$. An element of $\mathscr{D}_{S}$ for any such $S$ is called a special dendron. Two special dendrons $D_{\mathbf{s}}$ and $D_{2}$ are called similar if and only if there exists a class $\mathscr{D}_{S}$ to 
which they both belong.

Lemma 2.1. Let $D$ be a dendron and let $B$ be an order basis: for $D$ containing all the cut points of order greater than or equal. to three. Then for each $\varepsilon>0$, there exists a regular $\varepsilon$-cover with respect to $B$.

Proof. See Lemma 4.1 of [2].

Lemma 2.2. Let $X$ and $Y$ be dendrons and let $\varepsilon_{i} \rightarrow 0$. Let $\left\{\mathscr{U}_{i}\right\}_{i=1}^{\infty}$ and $\left\{\mathscr{V}_{i}\right\}_{i=1}^{\infty}$ be sequences of closed covers of $X$ and $Y$ respectively, such that

(1) $\mathscr{U}_{i}$ is a regular $\varepsilon_{i}$-cover of $X$,

(2) $\mathscr{V}_{i}$ is a regular $\varepsilon_{i}$-cover of $Y$,

(3) $\mathscr{U}_{i+1}$ is a refinement of $\mathscr{U}_{i}$; $\mathscr{V}_{i+1}$ is a refinement of $\mathscr{V}_{i}$,

(4) $\mathscr{Q}_{i}$ and $\mathscr{V}_{i}$ have the same number of elements,

(5) The elements of $\mathscr{U}_{i}$ and $\mathscr{V}_{i}^{i}$ can be so named that $U_{i_{j}} \cap U_{i_{k}} \neq \varphi$. if and only if $V_{i_{j}} \cap V_{i_{k}} \neq \varphi$,

(6) $U_{i+1, j} \leqq U_{i, k}$ if and only if $V_{i+1, j} \leqq V_{i, k}$.

Then $X$ and $Y$ are homeomorphic.

Proof. Let $x \in X$. Then there exists a tower of sets $\left\{U_{i, j_{x}}\right\}_{i=1}^{\infty}$ such that $x=\bigcap_{i=1}^{\infty} U_{i, j_{x}}$. Let $h(x)=\bigcap_{i=1}^{\infty} V_{i, j_{x}}$. It may be shown, by a standard argument, that $h(x)$ is well-defined, and is a homeomorphism of $X$ onto $Y$. (See, for example, Theorem 11 of [1].)

Lemma 2.3. Let $D$ be a dendron and let $\mathscr{U}: U_{1}, U_{2}, \cdots, U_{n}$ be a regular $\varepsilon$-cover. Let $P$ be the set of points of intersection of the various elements of the cover; that is, $p \in P$ if and only if there exist $i, j$ such that $U_{i} \cap U_{j}=p$. Then the elements of $P$ can be so named that $D-\left\{p_{1}\right\}$ has exactly one component which meets $P-\left\{p_{1}\right\}$, if this set is not empty.

Proof. Let $p_{1}^{\prime}$ be any element of $P$. If there exist at least two components of $D-\left\{p_{1}^{\prime}\right\}$ which contain elements of $P-\left\{p_{1}^{\prime}\right\}$, let $V_{1}$ be one of these components, and consider the points of $P$ in $V_{1}$. Let $p_{2}^{\prime}$ be any such point of $P$. Now consider $D-\left\{p_{2}^{\prime}\right\}$. One component of this set contains $p_{1}^{\prime}$. If no other components contain points of $P$, then let $p_{2}^{\prime}=p_{1}$, and we are through. If there are other components containing points of $P$, let $V_{2}$ be such a component. We note that $p_{1}^{\prime} \notin V_{2}$. Let $p_{3}^{\prime}$ be any element of $P$ in $V_{2}$. Consider $D-\left\{p_{3}^{\prime}\right\}$. We note that $p_{1}^{\prime}$ and $p_{2}^{\prime}$ are in the same component of this set, since $p_{2}^{\prime}$ separates $p_{1}^{\prime}$ from $p_{3}$. If this is the only component containing points of $P$, let $p_{3}^{\prime}=p_{1}$, and we are through. If there are other components containing points of $P$, we continue the above process. This process 
can be continued only a finite number of times, since $P$ is finite. Therefore, at the last stage we have the required $p_{1}$.

Lemma 2.4. Let $X$ and $Y$ be similar dendrons, and let $\varepsilon>0$. Let $A$ and $B$ be order bases for $X$ and $Y$ respectively, each containing all the cut points of order greater than or equal to three. Then $X$ and $Y$ admit regular $\varepsilon$-covers $\mathscr{Q}$ and $\mathscr{Y}$ with respect to $A$ and $B$ respectively, such that:

(1) $\mathscr{C}$ and $\mathscr{V}$ have the same number of elements, and

(2) the elements of $\mathscr{Q}$ and $\mathscr{V}^{\wedge}$ can be so named that $U_{i} \cap U_{j} \neq \varphi$ if and only if $V_{i} \cap V_{j} \neq \varphi$.

Proof. Let $\mathscr{C}^{\prime}: U_{1}^{\prime}, U_{2}^{\prime}, \cdots, U_{r}^{\prime}$ be a regular $\varepsilon$-cover of $X$ with respect to $A$. We may assume, without loss of generality, that the interiors of the elements of $\mathscr{C}^{\prime}$ are connected. Let $y_{0}$ be an endpoint of $Y$, and let $W_{1}$ be a connected neighborhood of $y_{0}$, of diameter less than $\varepsilon$. Let $y_{1}$ be a point of order two in $W_{1}$. Then $y_{1}$ is a point of order two in $Y$, and one of the two components of $Y-\left\{y_{1}\right\}$ is of diameter less than $\varepsilon$. Call this component $W_{2}$. Let $W_{2}^{\prime}$ be the other.

We will put a copy of $\mathscr{C}^{\prime}$ in $\bar{W}_{2}$ and a copy of a refinement of $\bar{W}_{2}^{\prime}$ in an appropriate element of $\mathscr{Q}^{\prime}$ to obtain the isomorphic covers $\mathscr{C}$ and $\mathscr{Y}$ of the theorem. We do this in the following way. Let $P$ be the set of points of $X$ which are points of intersection of the elements of $\mathscr{Z}^{\prime}$; that is, $p \in P$ if and only if there exists $U_{i}^{\prime}$ and $U_{j}^{\prime}$ such that $U_{i}^{\prime} \cap U_{\jmath}^{\prime}=p$. By Lemma 2.3, there exists $p_{1} \in P$ such that $X-\left\{p_{1}\right\}$ contains exactly one component meeting $P-\left\{p_{1}\right\}$. Let $k_{1}$ be the order of $p_{1}$. Let $q_{1}$ be a point of $W_{2}$ of order $k_{1}$. There are $\left(n_{1}-1\right)$ components of $X-\left\{p_{1}\right\}$ which do not contain elements of $P-\left\{p_{1}\right\}$. Let $\varphi_{1}$ assign the closures of these in any one-to-one manner to the closures of the $\left(n_{1}-1\right)$ components of $W_{2}-\left\{q_{1}\right\}$ which do not contain $y_{1}$. Let $O_{1}$ and $O_{1}^{\prime}$ be the remaining components of $X-\left\{p_{1}\right\}$ and $\bar{W}_{2}-\left\{q_{1}\right\}$ respectively, and let $\varphi_{1}$ assign $\bar{O}_{1}$ to $\bar{O}_{1}^{\prime}$.

We next break up $\bar{O}_{1}^{\prime}$ to match $\bar{O}_{1}$. $\bar{O}_{1}$ is a dendron containing $p_{1}$ as an endpoint, and $\bar{O}_{1}^{\prime}$ is a dendron containing $q_{1}$ as an endpoint. $\bar{O}_{1}$ already has inherited a regular $\varepsilon$-cover by elements of $\mathscr{Q}^{\prime}$. By Lemma 2.3, there exists $p_{2} \in P$ such that $\bar{O}_{1}-\left\{p_{2}\right\}$ has exactly one component which contains points of $P-\left\{p_{1}, p_{2}\right\}$, if this set is not empty. Let $k_{2}$ be the order of $p_{2}$. Let $q_{2}$ be a point of $\bar{O}_{1}^{\prime}$ of order $k_{2}$. Exactly one component of $\bar{O}_{1}-\left\{p_{2}\right\}$ contains $p_{1}$. Let $\varphi_{2}$ assign this component to the component of $\bar{O}_{1}^{\prime}-\left\{q_{2}\right\}$ which contains $q_{1}$. Let $\varphi_{2}$ map the closures of the other components arbitrarily in a one-toone manner to the closures of the components of $\bar{O}_{1}^{\prime}-\left\{q_{2}\right\}$ which do not contain $q_{1}$. We will call the component of $\bar{O}_{1}-\left\{p_{2}\right\}$ which con- 
tains points of $P-\left\{p_{1}, p_{2}\right\}$ by the name $O_{2}$, and we will call $\varphi_{2}\left(\bar{O}_{2}\right)$ by the name $\bar{O}_{2}^{\prime}$.

We note that $O_{2}$ has at most two boundary points in $P$. We next break up $\bar{O}_{2}^{\prime}$. By Lemma 2.3, there exists $p_{3} \in O_{2}$ such that $\bar{O}_{2}-\left\{p_{3}\right\}$ has exactly one component which meets $P-\left\{p_{1}, p_{2}, p_{3}\right\}$, if this set is not empty. Let $k_{3}$ be the order of $p_{3}$. We choose a point $q_{3}$ in $\bar{O}_{2}^{\prime}$ such that (1) the order of $q_{3}$ is $k_{3}$, and (2) $q_{i}$ separates $q_{j}$ from $q_{k}$ if and only if $p_{i}$ separates $p_{j}$ from $p_{k}, i, j, k=1,2,3$. In case $p_{i}$ does not separate $p_{j}$ from $p_{k}, i, j, k=1,2,3$, let $A_{1}$ be the unique arc from $p_{1}$ to $p_{2}$, and let $A_{1}^{\prime}$ be the unique are from $p_{3}$ to $A_{1}$. Let $k_{3}^{\prime}$ be the order of point $A_{1} \cap A_{1}^{\prime}$. In this case, we additionally require that $q_{3}$ have the corresponding property; that is, if $B_{1}$ is the arc from $q_{1}$ to $q_{2}$ and $B_{1}^{\prime}$ is the arc from $q_{3}$ to $B_{1}$ then the order of the point $B_{1} \cap B_{1}^{\prime}$ is also $k_{3}^{\prime}$. Now $\bar{O}_{2}-\left\{p_{3}\right\}$ has exactly one component which meets $P-\left\{p_{1}, p_{2}, p_{3}\right\}$, if this set is not empty. Look at the boundary points of this component, and let $\phi_{3}$ take this component to the component of $\bar{O}_{2}^{\prime}-\left\{q_{3}\right\}$ with the corresponding boundary points. Let $\varphi_{3}$ map the closures of the remaining components arbitrarily to the closures of the remaining components of $\bar{O}_{2}^{\prime}-\left\{q_{3}\right\}$, except that if one of the components of $\bar{O}_{2}-\left\{p_{3}\right\}$ has $p_{1}$ (or $p_{2}$ ) as an additional boundary point, let $\varphi_{3}$ take the closure of this component to the closure of the component of $\bar{O}_{2}^{\prime}-\left\{q_{3}\right\}$ with $q_{1}$ (or $q_{2}$ ) as an additional boundary point.

We continue this process inductively, choosing $q_{n}$ so that $q_{n}$ separates $q_{i}$ from $q_{j},(i, j<n)$, if and only if $p_{n}$ separates $p_{i}$ from $p_{j}$. Further, we ask that if the component of $X-\left\{p_{1}, p_{2}, \cdots, p_{n-1}\right\}$ which contains $p_{n}$ has $p_{i}(i<n)$ as a boundary point, then the component of $W_{2}-\left\{q_{1}, q_{2}, \cdots q_{n-1}\right\}$ which contains $q_{n}$ has $q_{i}$ as a boundary point. We also require that the order of $q_{n}$ equal the order of $p_{n}$. In case $p_{n}$ does not separate any pair $p_{i} p_{j}, 1 \leqq i, j \leqq n-1$, let $A_{n}$ be the union of the arcs joining the pairs of points $p_{i}, p_{j}$ for $1 \leqq i, j \leqq n-1$. Let $A_{n}^{\prime}$ be the arc joining $p_{n}$ to $A_{n}$. Let the order of the point $A_{n} \cap A_{n}^{\prime}$ be $k_{n}^{\prime}$. In this case we additionally require that $q_{n}$ have the corresponding property; that is, if $B_{n}$ is the union of the arcs joining $q_{i}$ and $q_{j}, 1 \leqq i, j \leqq n-1$, and if $B_{n}^{\prime}$ is the arc from $q_{n}$ to $B_{n}$. Then (a) the order of the point $B_{n} \cap B_{n}^{\prime}$ is $k_{n}^{\prime}$, and (b) $A_{n}^{\prime}$ and $B_{n}^{\prime}$ are isomorphic trees, the isomorphism determined by $p_{i} \rightarrow q_{i}$. We define $\varphi_{n}$ from the set of closures of components of $\bar{O}_{n-1}-\left\{p_{n}\right\}$ to the set of closures of components of $\bar{O}_{n-1}^{\prime}-\left\{q_{n}\right\}$ so that if $\varphi_{n}(\bar{O})=\bar{O}^{\prime}$ and $p_{i}$ is a boundary point of $\bar{O}$, then $q_{i}$ is a boundary point of $\bar{O}^{\prime}$.

After a finite number of steps, we use the last $p_{i}$ in $P$. Let $\phi$ be the one-to-one function determined by $\varphi_{1}, \varphi_{2}, \cdots, \varphi_{k}$, where $k$ is the number of points in $P$, and $\varphi\left(U^{\prime}\right)=\varphi_{j}\left(U^{\prime}\right)$ for some $j$ such that $U^{\prime}$ contains no points of $P$ in the $j$ th step of the above process, and where $U^{\prime} \in \mathscr{U}^{\prime}$. 
Let $\varphi\left(\bar{U}_{i}^{\prime}\right)=\bar{V}_{i}^{\prime}$ in $\bar{W}_{2}$, and call this cover of $\bar{W}_{2}$ by the name $\mathscr{V}^{\prime}$. Let $\bar{V}_{s}^{\prime}$ be the element of $\mathscr{V}^{\prime}$ which contains $y_{1}$. Consider $\varphi^{-1}\left(\bar{V}_{s}^{\prime}\right)=\bar{U}_{s}^{\prime}$. If $\bar{U}_{s}^{\prime}$ is an "end element" of $\mathscr{C}^{\prime}$, that is, the boundary of $\bar{U}_{s}^{\prime}$ contains exactly one point in $P$, let $x_{1}$ be any point of order two in $\bar{U}_{s}^{\prime}$, and let $W_{2}^{\prime \prime}$ be the component of $X-\left\{x_{1}\right\}$ which does not contain the points of $P$.

If $U_{s}^{\prime}$ is not an end element, let $Q=\left\{q_{i} \mid q_{i}\right.$ corresponds to $p_{i}$ in $P$, and let $B$ the finite tree which is the union of arcs joining the points of $Q$ on the boundary of $\bar{V}_{s}^{\prime}$, and let $B^{\prime}$ be the arc joining $y_{1}$ to $B$. Let $y=B \cap B^{\prime}$ and let $k$ be the order of $y$. Note that $k \geqq 3$. Now let $A$ be the finite tree which is the union of the arcs joining the points of $P$ on the boundary of $U_{s}^{\prime} . \quad A$ is isomorphic to $B$. Choose a point $x \in A$, of order $k$, such that $x$ separates $p_{i}$ from $p_{j}$ in $A$ if and only if $y$ separates $q_{i}$ from $q_{j}$ in $B$. Let $x_{1}$ be any point of order 2 of a component of $A-\{x\}$ whose only boundary point is $x$. Let $W_{2}^{\prime \prime}$ be the component of $X-\left\{x_{1}\right\}$ which does not contain points of $P$.

Now let $\mathscr{E}^{\prime}$ be a regular $\varepsilon$-cover of $W_{2}^{\prime}$. Let $\mathscr{E} "$ "be a copy of $\mathscr{E}^{\prime}$ in $W_{2}^{\prime \prime}$, using the same procedure as above, and making sure in the first step that the component in $W_{2}^{\prime}$ which contains $y_{1}$ corresponds to the component in $W_{2}^{\prime \prime}$ which contains $x_{1}$. $\mathscr{E}^{\prime \prime}$ is obtained in a finite number of steps. Let $\psi: \mathscr{E}^{\prime} \rightarrow \mathscr{E}^{\prime \prime}$ be the one-to-one function obtained.

The subdivisions $\mathscr{U}$ and $\mathscr{Y}$ of $X$ and $Y$ obtained in this manner are regular $\varepsilon$-covers of $X$ and $Y$ respectively, and are isomorphic; that is, there is a one-to-one function $f: \mathscr{U} \rightarrow \mathscr{V}^{-}$such that $U_{i} \cap U_{j} \neq \varphi$ if and only if $f\left(U_{i}\right) \cap f\left(U_{j}\right) \neq \varphi$. This completes the proof.

THEOREM 2.1. Let $X$ and $Y$ be two similar dendrons containing endpoints $\{a, b\}$ and $\{c, d\}$ respectively. Then there exists a homeomorphism $h, h: X \rightarrow Y$ such that $h(\alpha)=c$ and $h(b)=d$.

Proof. Let $\varepsilon>0$. We show that there exist regular $\varepsilon$-covers $\mathscr{U}$ and $\mathscr{V}$ for $X$ and $Y$ respectively, and a one-to-one function $\varphi$ from $\mathscr{U}$ onto $\mathscr{V}$ such that (1) $U_{i} \cap U_{j} \neq \varphi$ if and only if

$$
\varphi\left(U_{i}\right) \cap \varphi\left(U_{j}\right) \neq \varphi \text {, }
$$

and (2) $\varphi$ takes the element of $\mathscr{W}$ containing " $a$ " to the element of $\mathscr{V}$ containing " $c$," and the element of $\mathscr{U}$ containing " $b$ " to the element of $\mathscr{Y}$ containing " $d . "$

Let $x_{1}$ be a point of order two separating $a$ from $b$ in $X$, and let $A_{1}$ and $B_{1}$ be the two components of $X-\left\{x_{1}\right\}$. Let $y_{1}$ be a point of order two separating $c$ from $d$ in $Y$, and let $C_{1}$ and $D_{1}$ be the two components of $Y-\left\{y_{1}\right\}$. Let $\mathscr{U}^{\prime}$ be a regular $\varepsilon$-cover of $X$ which is 
the union of a regular $\varepsilon$-cover of $A_{1}$ and a regular $\varepsilon$-cover of $B_{1}$. Let $\mathscr{V}^{\prime \prime}$ be a regular $\varepsilon$-cover of $Y$ such that $\mathscr{V}^{\prime \prime}$ is the union of a regular $\varepsilon$-cover of $C_{1}$ and a regular $\varepsilon$-cover of $D_{1}$ and such that there exists a one-to-one function $\varphi: \mathscr{U}^{\prime} \rightarrow \mathscr{Y}^{\prime \prime}$, constructed with care as in Lemma 2.4, so that:

(1) $\varphi$ takes each element containing $x_{1}$ to an element containing $y_{1}$;

(2) $\varphi$ takes each element which is a subset of $A_{1}$ to an element which is a subset of $C_{1}$, and each element which is a subset of $B_{1}$ to an element which is a subset of $D_{1}$, and

(3) $\varphi$ takes the element containing " $a$ " to the element containing " $c$," and the element containing " $b$ " to the element containing " $d$."

Now let $\varepsilon_{i} \rightarrow 0$. For each $\varepsilon_{i}$ we construct isomorphic covers $\mathscr{U}_{i}$ and $\mathscr{V}_{i}$ with properties of $\mathscr{U}$ and $\mathscr{V}^{-}$above, and such that (1) $\mathscr{U}_{i}$ is a refinement of $\mathscr{U}_{i-1}$ and $\mathscr{V}_{i}$ is a refinement of $\mathscr{V}_{i-1}$, and (2) $U_{i j} \subseteq$ $U_{i-1, k}$ if and only if $V_{i j} \leqq V_{i-1, k}$. Clearly this may be done by using the care used in the construction of the isomorphic covers of Lemma 2.4 , and applying this to each element of $\mathscr{C}_{i-1}$, when constructing $\mathscr{C}_{i}$. Then it follows from Lemma 2.2 that there is a homeomorphism $h$, $h: X \rightarrow Y$ such that $h(\mathrm{a})=b$ and $h(c)=d$.

3. The main theorem. In this section we show that the space of homeomorphisms of a special dendron is zero dimensional and nowhere discrete. Thus it is not locally contractible.

Definition 3.1. A topological space is called contractible if and only if there exists a continuous function $F: X \times I \rightarrow X$ such that

(1) $F(x, 0)=x$, and

(2) $F(x, 1)$ is constant, for all $x \in X$.

Definition 3.2. $X$ is called locally contractible if and only if for each $x \in X$ and neighborhood $U$ of $x$, there are a neighborhood $V$ of $x$ and a continuous function $F: V \times I \rightarrow U$ such that

(1) $F(v, 0)=v$, and

(2) $F(v, 1)$ is constant, for some constant in $U$, all $v \in V$.

REMARK. A dendron is a locally connected continuum. Therefore a component $V$ of any open set $U$ is also open. Further, its closure is again a dendron. It is well-known that a dendron is contractible (See [3]), and thus it is also locally contractible.

THeorem 3.1 Let $X$ be a special dendron. Then $G(X)$ is nowhere discrete. 
Proof. It is sufficient to show that the identity is not isolated. Let $\varepsilon>0$. We show there exists an $h \in G(X), h \neq e$, such that $d(h, e)<\varepsilon$. Let $a, b$ be two endpoints of $X$, and let $U$ be a connected neighborhood of $b$ of diameter less than $\varepsilon$. Let $p_{1}, p_{2}, p_{3}, p_{4}$ be points of $U$ of order two such that $p_{1}$ separates $a$ from $b, p_{2}$ separates $p_{1}$ from $b, p_{2}$ separates $p_{2}$ from $b$, and $p_{4}$ separates $p_{3}$ from $b$. Thus the unique arc from $a$ to $b$ contains these points in the order $a-p_{1}-p_{2}-p_{3}-p_{4}-b$. Exactly one component of $X-\left\{p_{1}, p_{3}\right\}$ contains $p_{2}$. Call the closure of this component $A$. Exactly one component of $X-\left\{p_{1}, p_{4}\right\}$ contains $p_{2}$ (and $p_{3}$ ). Call the closure of this component $A^{\prime}$. Exactly one component of $X-\left\{p_{3}\right\}$ contains $p_{4}$ (and $b$ ). Call the closure of this component $B$. Exactly one component of $X-\left\{p_{4}\right\}$ contains $b$. Call the closure of this component $B^{\prime}$. Let $h$ be a homeomorphism of $X$ onto itself which carries $A$ onto $A^{\prime}$ so that $h\left(p_{1}\right)=p_{1}$ and $h\left(p_{3}\right)=p_{4}$, and which carries $B$ to $B^{\prime}$ so that $h\left(p_{3}\right)=p_{4}$ and $h(b)=b$. Let $h$ be the identity on $X-(A \cup B)$. By Theorem 2.1, such a homeomorphism exists. Since $h$ moves only points of $A \cup B$, and $A \cup B \subseteq U$, and $\operatorname{diam} U<\varepsilon$, we know that $d(h, e)<\varepsilon$.

Theorem 3.2. Let $X$ be a special dendron. Then $G(X)$ is zero dimensional and is not locally contractible.

Proof. Since $X$ is special, the cut points of order greater than two are dense in $X$. Thus it follows from Theorem 4.1 of [2] that $G(X)$ is zero dimensional, and therefore no ares exist in $G(X)$. But if $G(X)$ were locally contractible, there would exist a continuous function $F: U \times I \rightarrow G(X)$ which is not constant on $\{g\} \times I$, for some $g \in U$, where $U$ is some open set in $G(X)$. But $F \mid\{g\} \times I$ is a nonconstant continuous function from an are into $G(X)$, and therefore must contain an arc. This is a contradiction. It follows that $G(X)$ is not locally contractible.

\section{BIBLIOGRAPHY}

1. R. H. Bing, A homogeneous indecomposable plane continuum. Duke Math. J. 15, (1948), 729-742.

2. B. Brechner, On the dimensions of certain spaces of homeomorphisms. Trans. Amer. Math. Soc. 121 (1966), 516-48.

3. Karl Menger, Kurventheorie. Teubner, Leipzig and Berlin, 1932.

4. G. T. Whyburn, Analytic topology. AMS colloquium Publications, Vol. 28, Amer. Math. Society, Providence, R. I, 1942; reprint 1955.

Received January 30, 1968, and in revised form June 3, 1968.

UNIVERSITY OF FLORIDA

Gainesville, Florida 



\section{PACIFIC JOURNAL OF MATHEMATICS}

\section{EDITORS}

H. ROYDEN

Stanford University

Stanford, California

\section{R. $R$ PHELPS}

University of Washington

Seattle, Washington 98105

\section{J. DugunduI}

Department of Mathematics

University of Southern California

Los Angeles, California 90007

RICHARD ARENS

University of California

Los Angeles, California 90024

\section{ASSOCIATE EDITORS}

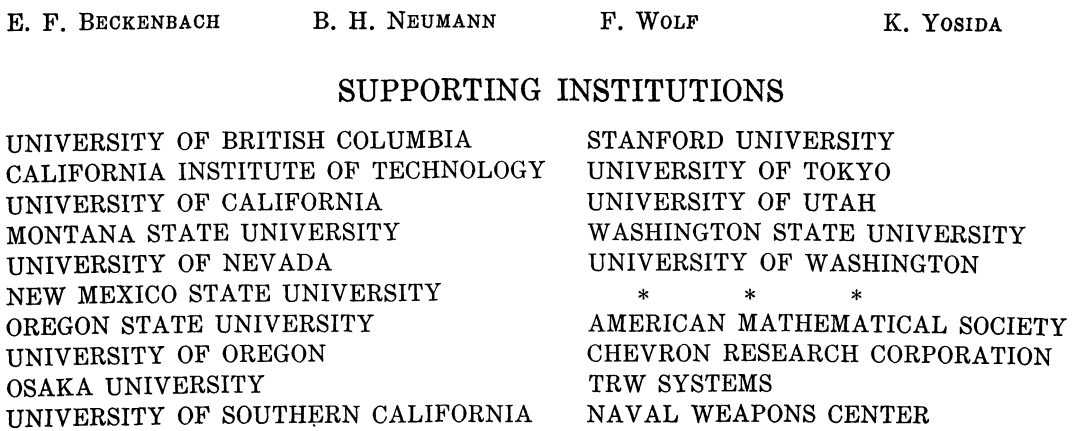

The Supporting Institutions listed above contribute to the cost of publication of this Journal, but they are not owners or publishers and have no responsibility for its content or policies.

Mathematical papers intended for publication in the Pacific Journal of Mathematics should be in typed form or offset-reproduced, double spaced with large margins. Underline Greek letters in red, German in green, and script in blue. The first paragraph or two must be capable of being used separately as a synopsis of the entire paper. It should not contain references to the bibliography. Manuscripts, in duplicate if possible, may be sent to any one of the four editors. Please classify according to the scheme of Math. Rev. 36, 1539-1546. All other communications to the editors should be addressed to the managing editor, Richard Arens, University of California, Los Angeles, California, 90024.

50 reprints are provided free for each article; additional copies may be obtained at cost in multiples of 50 .

The Pacific Journal of Mathematics is published monthly. Effective with Volume 16 the price per volume (3 numbers) is $\$ 8.00$; single issues, $\$ 3.00$. Special price for current issues to individual faculty members of supporting institutions and to individual members of the American Mathematical Society: $\$ 4.00$ per volume; single issues $\$ 1.50$. Back numbers are available.

Subscriptions, orders for back numbers, and changes of address should be sent to Pacific Journal of Mathematics, 103 Highland Boulevard, Berkeley, California, 94708.

PUBLISHED BY PACIFIC JOURNAL OF MATHEMATICS, A NON-PROFIT CORPORATION

Printed at Kokusai Bunken Insatsusha (International Academic Printing Co., Ltd.), 7-17, Fujimi 2-chome, Chiyoda-ku, Tokyo, Japan. 


\section{Pacific Journal of Mathematics}

Vol. 28, No. $2 \quad$ April, 1969

Richard Arens and Donald George Babbitt, The geometry of relativistic

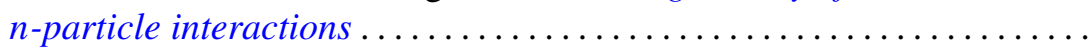

Kirby Alan Baker, Hypotopological spaces and their embeddings in lattices

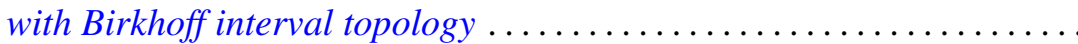

J. Lennart (John) Berggren, Finite groups in which every element is conjugate to its inverse ........................... 289

Beverly L. Brechner, Homeomorphism groups of dendrons . . . . . . . . . . . 295

Robert Ray Colby and Edgar Andrews Rutter, QF - 3 rings with zero singular ideal ................................. 303

Stephen Daniel Comer, Classes without the amalgamation property....... 309

Stephen D. Fisher, Bounded approximation by rational functions ......... 319

Robert Gaines, Continuous dependence for two-point boundary value problems..................................... 327

Bernard Russel Gelbaum, Banach algebra bundles ............... 337

Moses Glasner and Richard Emanuel Katz, Function-theoretic degeneracy criteria for Riemannian manifolds ...................... 351

Fletcher Gross, Fixed-point-free operator groups of order $8 \ldots \ldots \ldots \ldots 357$

Sav Roman Harasymiv, On approximation by dilations of distributions . . . . 363

Cheong Seng Hoo, Nilpotency class of a map and Stasheff's criterion ... . . 375

Richard Emanuel Katz, A note on extremal length and modutus.......... 381

H. L. Krall and I. M. Sheffer, Difference equations for some orthogonal polynomials ................................

Yu-Lee Lee, On the construction of lower radical properties ........... 393

Robert Phillips, Liouville's theorem........................... 397

Yum-Tong Siu, Analytic sheaf cohomology groups of dimension $n$ of

n-dimensional noncompact complex manifolds ..... . .

Michael Samuel Skaff, Vector valued Orlicz spaces. II...

James DeWitt Stein, Homomorphisms of $B^{*}$-algebras .... . .

Mark Lawrence Teply, Torsionfree injective modules .... . . .

Richard R. Tucker, The $\delta^{2}$-process and related topics. II .

David William Walkup and Roger Jean-Baptiste Robert Wets, Lifting

projections of convex polyhedra...

Thomas Paul Whaley, Large sublattices of a lattice. 